\title{
The satisfaction level of the pain treatment with the America Pain Society - Patient Outcome Questionnaire
}

\author{
Romeo A., C. Mamazza, E. Cristaldi, R. Risicato \\ U.O.C. Medicina Interna P.O. «Muscatello» Asp Siracusa
}

Background: For some time now we have been talking about hospital without pain, and there are various scales used to evaluate it.

The aim of the study is to evaluate the patient's perceived satisfaction with the treatment of pain in relation to the care received.

Methods: We used the American Pain Society - Patient Outcome Questionnaire (APS-POQ) proved valid and reliable even in Italian. An easy-to-impart tool consisting of 16 items provided by the psychologist to the patients through an interview. This project analyzed a sample of 35 hospitalized patients, aged between 48-82 years.

Patients with cognitive deficits, psychiatric or psychophysical that do not allow the questionnaire completion were excluded. The aim was to verify the presence of pain in its intensity and impact in different areas of activity; patient satisfaction for pain management and reasons for satisfaction/dissatisfaction; the presence of erroneous pain management beliefs.

Results: Only $12 \%$ of the patients reported mild pain, $19 \%$ moderate, $12 \%$ had no pain. The reported average intensity of pain was $6 / 10(47 \%)$. The highest pain intensity of hospitalization was $8 / 10(10 \%)$. Patients reported the highest level of pain impact on general activities while the lowest level on walking.

Although most patients reported that their pain was adequately controlled, $18 \%$ of them instead considered their pain poorly controlled.

The satisfaction with the treatment of pain was positive both for the modalities with which the nurses and the doctors responded.

Discussion: To the question "Did you feel pain in the last 24 hours?» Only 33\% answered Yes but all the patients had pain relief treatment (Table 1).

Very often the patients were not able to define the etiology of pain or the exact location if occurred in the days before the interview.

The characteristics of the patients can certainly influence the level of pain experienced however pain is also common in hospitalized patients at lower risk and therefore each person must be considered at high risk.

Conclusion: In the future it would be advisable not only to check the effectiveness of the treatment of pain but also the patient's perception. It would be a further indicator of the quality of treatment as a feedback of the interventions carried out. 